\title{
REHABILITACIÓN Y TERAPIA OCUPACIONAL DESDE UN ENFOQUE CENTRADO EN LA PERSONA Y BASADO EN LA OCUPACIÓN. UN ESTUDIO DE CASO DE ARTRITIS PSORIÁSICA
}

\author{
REHABILITATION AND OCCUPATIONAL THERAPY FROM A PERSON-CENTRED AND \\ OCCUPATION-BASED APPROACH. A CASE STUDY OF PSORIATIC ARTHRITIS.
}

\section{Nerina Soledad Zisaํㅜ Cesar Rubio Belmonte ${ }^{2}$}

\begin{abstract}
RESUMEN
Se presenta un estudio de caso con diagnóstico de artritis psoriásica. La propuesta de intervención se articuló desde un enfoque centrado en la persona y basado en las necesidades ocupacionales, atendiendo al posicionamiento institucional de la Federación Mundial de Terapeutas Ocupacionales (WFOT).

Objetivo: Evaluar la eficacia de un programa de terapia ocupacional individualizado basado en la ocupación y centrado en un paciente con artritis psoriásica, en términos de mejoría en el desempeño ocupacional y la calidad de vida percibida.

Metodología: Se evaluaron problemas del desempeño ocupacional (COPM y DASH), así Como la calidad de vida percibida (WHOQOL-BREF). Secundariamente se registraron medidas de dolor (EVA), movilidad articular (goniometría y mediciones centimétricas) y fuerza (dinamometría). Se establecieron objetivos funcionales de intervención desde la metodología SMART y se aplicó un programa de intervención combinando diversas modalidades de tratamiento que vienen acumulando diversos niveles de evidencia científica: férulas, productos de apoyo, ejercicio, protección articular yeducación para la salud.

Resultados: Se registraron mejoras significativas en desempeño ocupacional (COPM y GAS), así como en satisfacción (COPM) y en calidad de vida percibida (WHOQOL-BREF). Se obtuvieron, igualmente, resultados positivos en el resto de las variables observadas: reducción del dolor, incremento del movimiento articular y de la fuerza muscular.

Conclusiones: La intervención propuesta, bajo un enfoque top-down logró cambios significativos no sólo en el desempeño ocupacional y en la calidad de vida percibida, sino también en las deficiencias de funciones corporales.
\end{abstract}

\section{PALABRAS CLAVE}

Artritis psoriásica; COPM; práctica centrada en la persona; terapia ocupacional; desempeño ocupacional; calidad de vida.

1 Graduada en Terapia Ocupacional por la Universidad Complutense de Madrid. Master en Terapia ocupacional basada en la evidencia. La Salle Centro Universitario. Institución de Afiliación Principal: Centro Logros ORCID: 0000-0003-2861-5368 Calle del Calvario 5, bajo 1, Madrid, 28012. Email: nerinazisa@gmail.com. 697715512.

2 Graduado en Terapia Ocupacional por la Universidad de Zaragoza. Doctor por el Programa de Psicología y Ciencias de la Salud de la Universidad Católica de Valencia Institución de Afiliación Principal: Universidad Católica de Valencia "San Vicente Mártir". ORCID: 00000003-0689-4984 Calle Guillem de Castro 175, Valencia, 46008. Email: cesar.rubio@ucv.es, 609243354. 


\begin{abstract}
A case study with a diagnosis of psoriatic arthritis is presented. The intervention proposal was articulated from a person-centered and occupational-based approach, attending to the position statement of the World Federation of Occupational Therapists (WFOT).

Aim: To evaluate the efficacy of an individualized occupational therapy program, based on occupation-based and centered on a patient with psoriatic arthritis, in terms of occupational performance improvement, and perceived quality of life.

Methodology: Occupational performance problems (COPM and DASH), as well as perceived quality of life (WHOQOL-BREF) were evaluated. Secondarily, pain measurements (VAS), joint mobility (goniometry and centimeter measurements) and strength (dynamometry) were recorded. Functional intervention aims were established from the SMART methodology, and an intervention program was applied combining various treatment modalities that have accumulated scientific evidence: splints, support products, exercise, joint protection and health education. Results: Significant improvements were registered in occupational performance (COPM and GAS), as well as in satisfaction (COPM) and in perceived quality of life (WHOQOL-BREF). Likewise, positive results were obtained in the rest of the variables observed: reduction in pain, increase in joint movement and muscle strength.

Conclusions: The proposed intervention, from a top-down approach, achieved significant changes not only in occupational performance and perceived quality of life, but also in deficiencies in body functions.
\end{abstract}

\title{
KEYWORDS
}

Psoriatic arthritis; COPM; person centred approach; occupational therapy.

Recibido 18/06/2019

Aceptado: 18/11/2020

\section{INTRODUCCIÓN}

La artritis psoriásica (en adelante APs) es una enfermedad autoinmune que forma parte de la clasificación de las espondiloartritis y que puede causar daño estructural progresivo en las articulaciones y discapacidad irreversible (Mease \& Coates, 2018). Las limitaciones funcionales causadas por la APs pueden llevar a un desequilibrio ocupacional viéndose significativamente afectadas las actividades de la vida diaria (en adelante AVD) (Kjeken et al., 2005), incidiendo directamente en el estado psicológico y funcional de la persona (Martínez Piédrola, Pérez de Heredia Torres, \& Gómez Calero, 2015).

La Federación Mundial de Terapeutas Ocupacionales (WFOT, 2019) en su documento de posicionamiento institucional con respecto a la terapia ocupacional (en adelante TO) y la rehabilitación enfatiza aspectos tales como: (1) la importancia de adoptar enfoques centrados en la persona, (2) dirigidos a la mejora del desempeño en los entornos en los que estos viven, trabajan y juegan; así como, (3) el compromiso con las ocupaciones ("engagement") que dotan de sentido a sus vidas. Todos estos aspectos vienen a delimitar claramente las bases teórico-conceptuales sobre las que deben sentarse las prácticas profesionales de rehabilitación en TO.

Por otro lado, los terapeutas ocupacionales en el ámbito de la rehabilitación, en general, y en el abordaje de las enfermedades reumáticas, en particular, están obligados a desarrollar sus intervenciones implementando modalidades de tratamiento que acumulen cierto nivel de evidencia (Ekelman et al., 2014). En este sentido, diversos trabajos de revisión han venido reportando diferentes niveles de evidencia para cinco grandes modalidades de tratamiento en el abordaje de la artritis: férulas (Steuljens et al., 2002; Ekelman et al., 2014), protección articular (Christie et al, 2007; Ekelman et al., 2014; Siegel, Tencza, Apodaca, \& Poole, 2017; Steuljens et al., 2002), ejercicios (Ekelman et al., 2014; Siegel et al., 2017), productos de apoyo (Steuljens et al., 2002) y educación para la salud (Christie et al, 2007; Ekelman et al., 2014; Siegel et al., 2017).

Siegel et al. (2017) concluyen en su trabajo de revisión sistemática que, pese a la existencia de evidencia con respecto a la efectividad de la TO en artritis reumatoide, escasas intervenciones están basadas en la ocupación. 
El objetivo del presente trabajo es evaluar la eficacia de un programa de TO individualizado basado en la ocupación y centrado en un paciente con artritis psoriásica, en términos de desempeño ocupacional y calidad de vida percibida, en el que se implementaron modalidades de tratamiento con respaldo de evidencia científica.

\section{MATERIAL y MéTOdo}

\section{Participante:}

Mujer de 25 años con diagnóstico de APs desde el 2014, derivada al departamento de TO de la Asociación de Artritis de Madrid. El motivo inicial de su consulta es la pérdida de fuerza y destreza bilateral, aunque refiere mayores dificultades en su mano dominante (derecha). Graduada en Bellas Artes, siendo su especialidad la escultura en piedra. Comenta que "mientras más duro el material más le gusta el tallado", pero desde que tiene la enfermedad y ha ido perdiendo fuerza en sus manos es consciente que debe cambiar de profesión. Está en búsqueda de trabajo activa en la rama de hostelería. Se muestra muy predispuesta a comenzar con el tratamiento y tiene buenas expectativas.

\section{Instrumentos:}

Canadian Occupational Performance Measure (COPM; Law et al., 1990). Se trata de una herramienta diseñada con el fin de favorecer la implementación de prácticas centradas en la persona desde una perspectiva ocupacional. Recoge y ordena, según la prioridad del paciente los problemas en el desempeño ocupacional en las áreas de autocuidado, productividad y ocio. Además, evalúa los cambios en la percepción del paciente sobre su desempeño ocupacional y la satisfacción con el mismo.

Disabilities of the Arm, Shouler and Hand (DASH; Mc Conell, Beaton y Bombardier, 1999). Se trata de un cuestionario autoadministrado, que permite valorar la discapacidad percibida por el paciente para realizar diversas actividades, incluidas actividades de la vida diaria y síntomas como el dolor, la rigidez o la pérdida de fuerza. Consta de 30 ítems y 2 módulos opcionales, con 4 ítems cada uno, destinados a medir el impacto de la lesión de miembro superior al tocar instrumentos musicales y al realizar deporte o trabajar. Cada ítem se puntúa de 1 a 5, con valores crecientes en función de la intensidad de los síntomas. La puntuación de los ítems se suma para obtener una puntuación total, que puede oscilar entre 30 y 150 puntos y que se transforma en una escala de o (mejor puntuación posible) a 100 (peor puntuación posible). En el presente trabajo se utilizó la validación española de Hervás el al., 2006.

Escala breve de Calidad de Vida de la oms (WHOQOL-BREF; WHOQOL Group, 1998). La WHOQOL-BREF es una escala autoadministrada, tipo Likert, con un total de 26 ítems que proporciona un perfil de la calidad de vida percibida por la persona en cuatro dimensiones: salud física (SF), salud psicológica (SP), relaciones sociales (RS) y ambiente (E). A mayores puntuaciones en cada una de las subescalas, mayor calidad de vida percibida. En el presente trabajo se utilizó la adaptación española de esta escala (Lucas-Carrasco, 2012).

Pese a no ser objetivo primario de este trabajo, que pretende estar centrado en la ocupación, se tomaron medidas de diversas deficiencias en funciones corporales relacionadas con la patología (Martínez Piédrola et al., 2015): (1) fuerza (dinamómetro de mano y de pinza), (2) dolor (escala visual analógica; EVA) y (3) movilidad articular (goniómetro y medidas centimétricas de oposición del pulgar). Todo ello bajo el supuesto de que las intervenciones centradas en la ocupación (enfoque top-down) muestran resultados óptimos relacionados en funciones corporales como fuerza y movimiento, en este caso.

\section{Procedimiento:}

Se llevó a cabo una sesión de evaluación dirigida a la recogida de datos mediante entrevista, exploración física y el pase de instrumentos/pruebas de evaluación arriba descritos.

Se establecieron seis objetivos de intervención centrados en la ocupación/funcionamiento derivados de los problemas descritos y priorizados por la paciente en la entrevista con la COPM. Los objetivos se redactaron atendiendo a la metodología SMART (Bovend'Eerdt, Botell, \& Wade, 2009). 
Se seleccionaron modalidades de tratamiento con evidencia científica: (1) participación en un programa de protección articular (en adelante PA), (2) TO individualizada dos veces por semana el primer mes y una vez por semana el siguiente mes (precalentamiento, terapia manual, ejercicios de flexibilidad y resistencia y AVD), (3) programa de ejercicios a domicilio, (4) confección de férulas rígidas de reposo nocturno y (5) consejo de productos de apoyo (en adelante PPAA) y adaptación del puesto de trabajo.

Se evaluaron los resultados de la intervención replicando el procedimiento de evaluación inicial y, además, se incluyó la Goal Attainment Scaling (GAS; Ottenbacher \& Cusick, 1990). para evaluar el grado de consecución de los objetivos.

Se recogió el consentimiento informado por parte del paciente, así como su autorización para la publicación de los resultados de la intervención implementada.

\section{Resultados}

\section{Resultados evaluación inicial}

Entrevista/anamnesis: la paciente refiere los siguientes síntomas y preocupaciones: (1) rigidez al sujetar utensilios como cubiertos o cepillo de dientes; (2) dificultad en la oposición del pulgar; (3) torpeza motora, se le caen las cosas de las manos y ha renunciado a ciertas actividades, como pintarse las uñas; (3) dedo en gatillo en $2^{\circ}$, $3^{\circ}, 4^{\circ}$ y $5^{\circ}$ dedos de ambas manos; (4) dolor continuo en sus manos y parestesias al coger objetos pequeños, pese a ello sigue intentando realizar todo tipo de gestos auto-instruyéndose con pensamientos tales como "el dolor será rápido"; (5) limitaciones leves en las actividades de aseo y vestido e importantes limitaciones en la preparación de alimentos; (6) estado anímico fluctuante, siendo totalmente consciente de la pérdida de fuerza, de la rigidez articular y de que la reducción de movimientos le impide continuar con sus AVD.

Entre los factores ambientales suponen un facilitador la circunstancia de contar con apoyo de una buena red de amistades y de su pareja. Por el contrario, son barreras o limitaciones: (1) el dolor no controlado, pese al tratamiento farmacológico; (2) el desconocimiento de las férulas y РPAA; $y$, (3) el desconocimiento de estrategias compensatorias tales como la PA o la conservación de energía (en adelante CE).

COPM: en el transcurso de la entrevista semiestructurada se identifican como prioritarias las necesidades y dificultades incluidas en la tabla 1:

$D A S H$ : la paciente obtuvo una puntuación total de 87 . Los resultados de la evaluación manifiestan dificultades para desarrollar y ejecutar actividades tales como: abrir botes, girar la llave, escribir, preparar la comida, empujar puertas, realizar las tareas duras de la casa, cargar bolsas u objetos pesados, utilizar cuchillo para cortar la comida y realizar actividades de entretenimiento.

WHOQOL-BREF: con una puntuación de 63, se manifiesta poca capacidad de concentración, insatisfacción a la hora de realizar las AVD, el trabajo y sueño, así como con las condiciones del hogar donde reside.

Resto de variables evaluadas: (1) dolor bilateral en ambas manos (4 - EVA); (2) limitación del rango activo de movimiento articular (AROM) en flexo-extensión de muñeca de $40^{\circ} / 20^{\circ}$ mano derecha y de $30^{\circ} / 40^{\circ}$ mano izquierda; (3) déficit en oposición del pulgar de $4,5 \mathrm{~cm}$ y $3,5 \mathrm{~cm}$ en manos derecha e izquierda, respectivamente; (4) reducción de la fuerza de prensión manual $3 \mathrm{~kg}$ y $8 \mathrm{~kg}$ en mano derecha e izquierda, respectivamente); (5) reducción de la fuerza de pellizco bidigital (okg en ambas manos), pellizco lateral (okg/ $1 \mathrm{~kg}$, mano derecha e izquierda) y pellizco tridigital (o, $5 \mathrm{~kg}$ en ambas manos).

\section{Resultados evaluación final}

En la tabla 2 se exponen los resultados de los objetivos funcionales tanto desde la perspectiva subjetiva de la paciente (COPM) como desde la perspectiva del profesional, a través de la Goal Attainment Scaling (GAS; Ottenbacher \& Cusick, 1990). En términos globales los cambios en el desempeño obtienen una puntuación de 6,34 y en la satisfacción con el desempeño una puntuación de 9, medidos a través de la CopM. Todos los objetivos trazados se han cumplido por encima de lo esperado, atendiendo a la evaluación mediante la GAS con puntuaciones de 1 y 2 en la totalidad de los objetivos.

En la tabla 3 se exponen los resultados relativos a las variables de discapacidad percibida (reducción), calidad 
TABLA 1 RESULTADOS EVALUACIÓN INICIAL COPM Y SINTOMATOLOGÍA ASOCIADA

\begin{tabular}{lll} 
Problema de Desempeño Ocupacional & Síntomas asociados $\quad \mathrm{DI}^{2} \quad \mathrm{Sl}^{3}$ \\
\hline
\end{tabular}

\section{Alimentación}

Cortar alimentos con cuchillo

Soltar los cubiertos

\section{Vestido}

Abrocharse el sujetador

Subirse las medias

Abrochar botones tipo clip

Subir cremalleras

\section{Cocina}

Cortar alimentos

Abrir botes

Abrir cafetera italiana

Sujetar vasos y platos

Trabajo

Búsqueda de empleo por internet

Lavado de ropa

Tender la ropa

Traslados en el interior del domicilio

Abrir con la llave

Girar los pomos de las puertas

Puntuaciones totales

30

Debilidad/Dolor

Rigidez

20

Rigidez

Debilidad

Destreza

Destreza

$1 \quad 0$

Debilidad

Debilidad

Debilidad

Debilidad

33

Dolor/Cansancio ${ }^{4}$

30

Debilidad

$4 \quad 0$

Debilidad

Debilidad

0 
TABLA 2 RESULTADOS EVALUACIÓN FINAL COPM Y ESTRATEGIAS DE INTERVENCIÓN IMPLEMENTADAS

\begin{tabular}{|c|c|c|c|c|}
\hline Problema de Desempeño Ocupacional & $\mathrm{DF}^{1}$ & $\mathbf{S F}^{2}$ & $\mathrm{GAS}^{3}$ & Estrategias de intervención \\
\hline \multirow[t]{2}{*}{ Alimentación } & 7 & 7 & 1 & Productos de apoyo \\
\hline & & & & Ergonomía (PA) \\
\hline \multirow[t]{2}{*}{ Vestido } & 9 & 10 & 2 & Productos de apoyo \\
\hline & & & & Ergonomía (PA) \\
\hline \multirow[t]{2}{*}{ Cocina } & 8 & 10 & 2 & Productos de apoyo \\
\hline & & & & Ergonomía (PA) \\
\hline \multirow[t]{2}{*}{ Trabajo } & 10 & 10 & 2 & Modificación entorno trabajo \\
\hline & & & & Ergonomía (PA/CE) \\
\hline \multirow[t]{2}{*}{ Lavado de ropa } & 10 & 10 & 2 & Productos de apoyo \\
\hline & & & & Ergonomía (PA/CE) \\
\hline Traslados en el interior del domicilio & 10 & 10 & 2 & Productos de apoyo \\
\hline Puntuaciones totales & 9 & 9,5 & & \\
\hline
\end{tabular}

Nota: (1) DF = Desempeño Final; (2) SF = Satisfacción Final; (3) Goal Attainment Scaling

TABLA 3 RESULTADOS DISCAPACIDAD PERCIBIDA, CALIDAD DE VIDA, FUERZA, DOLOR Y ROM

\begin{tabular}{llll}
\hline & PRE & POST & CAMBIOS \\
\hline DASH & 86,5 & 50 & $-36,5$ \\
WHOQOL-Bref & 63 & 80 & +17 \\
WHOQOL-Bref SF & 13 & 17 & +4 \\
WHOQOL-Bref SP & 18 & 20 & +2 \\
WHOQOL-Bref RS & 12 & 14 & +2 \\
WHOQOL-Bref E & 20 & 29 & +9 \\
Prensión mano* & $3 / 8 \mathrm{~kg}$ & $13 / 12 \mathrm{~kg}$ & $+10 /+4 \mathrm{~kg}$ \\
Prensión pellizco bidigital* & $0 / 0 \mathrm{~kg}$ & $3,5 / 3,5 \mathrm{~kg}$ & $+3,5 /+3,5 \mathrm{~kg}$ \\
Prensión pellizco lateral* & $0 / 1 \mathrm{~kg}$ & $3 / 3,5 \mathrm{~kg}$ & $+3 /+2,5 \mathrm{~kg}$ \\
Prensión pellizco tridigital* & $0,5 / 0,5 \mathrm{~kg}$ & $3,5 / 3,5 \mathrm{~kg}$ & $+3 /+3 \mathrm{~kg}$ \\
Dolor* & $4 / 4$ & $0 / 0$ & $-4 /-4$ \\
AROM Flexión muñeca* $^{*}$ & $40^{\circ} / 30^{\circ}$ & $55^{\circ} / 70^{\circ}$ & $+15^{\circ} /+40^{\circ}$ \\
AROM Extensión muñeca* $^{*}$ & $20^{\circ} / 40^{\circ}$ & $30^{\circ} / 40^{\circ}$ & $+10^{\circ} /=$ \\
Déficit oposición pulgar* $^{*}$ & $4,5 / 3,5 \mathrm{~cm}$ & $0 / 0 \mathrm{~cm}$ & $-4,5 /-3,5 \mathrm{~cm}$ \\
\hline Nota: Valores para mano & &
\end{tabular}

Nota: *Valores para mano derecha/mano izquierda 
de vida (incremento en puntuación total y en todas las dimensiones), fuerza (incremento), dolor (reducción) y rango de movimiento articular (incremento).

\section{Discusıón}

La liga europea contra el reumatismo (eULAR) afirma que una gestión óptima de la mano con osteoartritis requiere una combinación de tratamientos farmacológicos y no farmacológicos, recomendando un régimen de ejercicios que involucre el rango de movimiento, el fortalecimiento muscular y terapia ocupacional para mejorar las actividades, participación y calidad de vida (Zhang et al., 2007). Sin embargo, y pese a las recomendaciones de la EULAR y de la WFOT (2019), existen escasas referencias en la literatura de intervenciones basadas en la ocupación (Siegel et al., 2017) y que recojan resultados relativos al impacto de las intervenciones en la calidad de vida.

Con respecto a los resultados en el funcionamiento/ desempeño ocupacional en la literatura existen referencias que reportan óptimos resultados de la terapia ocupacional en la artritis reumatoide tanto en la mejora de los resultados funcionales (Riemsma, Taal, Kirwan \& Rasker, 2004; Steuljens, Dekker, Bouter, Leemrijse \& Van Der Ende, 2005) como en la reducción de la discapacidad (Riemsma et al., 2004). En el presente trabajo se evidencia una notable mejoría en todos los objetivos de intervención propuestos, por encima de los resultados esperados. Las puntuaciones de la сорм indican un cambio significativo en el desempeño y en la satisfacción con el desempeño desde el punto de vista del paciente. Se considera efectiva la intervención cuando los cambios en el desempeño o en la satisfacción son iguales o superiores a dos puntos (Law et al., 1990) y, en este caso, los cambios de desempeño han sido de 6,34 puntos y los cambios en la satisfacción con el desempeño de 9 puntos. Con respecto a la consecución de los objetivos funcionales propuestos, todas las puntuaciones de la GAS (Ottenbacher \& Cusick, 1990) indican que los objetivos se han logrado por encima de lo esperado. Además, se ha visto una reducción de la discapacidad percibida medida a través de la DASH (Hervás el al., 2006). Así pues, al finalizar el tratamiento la paciente no tiene percepción de dificultad para ejecutar actividades.

En relación a la calidad de vida percibida se aprecia una mejoría, tanto en las puntuaciones totales de la
WHOQOL-BREF (Lucas, 1998) como en cada una de las dimensiones medidas por esta escala, en este orden: salud física, entorno, salud psicológica y relaciones. Los resultados, más allá de las puntuaciones cuantitativas, se manifiestan en la satisfacción con los logros conseguidos expresada por la cliente y su entorno. Refiere ser capaz de continuar con sus AVD con mayor rendimiento y satisfacción, más allá de los objetivos planteados en la intervención. En la actualidad es capaz de conducir su coche, algo que no era una meta esperada por ella, dada su baja percepción de logro con respecto a la posibilidad de cumplir ese objetivo. Sus amigos, por su parte, comentan que vuelve a ser ella misma con sus uñas pintadas.

Pese a que para el planteamiento del caso se optó por un enfoque top-down y centrado en la ocupación, se encontraron resultados óptimos en los síntomas asociados al diagnóstico de la paciente y relacionados con su limitación funcional: dolor, debilidad y disminución del rango de movimiento articular. Siendo éstos, en síntesis, satisfactorios con un incremento de la fuerza de prensión en todas las variables medidas, desaparición del dolor, incremento de la movilidad activa en flexoextensión de la muñeca y oposición del pulgar. Estos resultados avalan la hipótesis de que un enfoque centrado en las limitaciones de las actividades también puede resultar en una mejoría de las funciones corporales.

El tratamiento desarrollado en este caso, se basó en la evidencia científica, tomando como principales modalidades de tratamiento la PA, férula de uso nocturno, PPAA y ejercicios. Haciendo especial hincapié en la PA y los ejercicios de manos ya que está demostrado que los mismos son efectivos a medio plazo (Dziedzic et al., 2015). Por lo general hay un alto nivel de evidencia que apoya los efectos de los ejercicios de la mano (Nivel 1A), no obstante, cuando se trata de buscar recomendaciones de ejercicios específicos, la evidencia está limitada a opiniones de expertos (Kjeken, 2011).

Finalmente, cabe destacar que diversos estudios demuestran la eficacia y la importancia que toma la educación terapéutica del paciente en el manejo de la artritis reumatoide (Christie et al., 2007; Hammond, Young \& Kidao, 2004; Siegel et al., 2017). Así pues, los programas de educación para la salud en artritis bajo un enfoque cognitivo-conductual, preferentemente, persiguen el empoderamiento de la persona para el cuidado de su propia salud. En este sentido, en el presente 
trabajo y tras la intervención implementada la cliente es consciente de que puede volver a tener nuevos brotes con repercusión en su capacidad funcional, pero a diferencia del pasado ahora se siente empoderada y manifiesta que dispone de las herramientas adecuadas para volver a superarlos.

\section{Limitaciones y proyecciones o recomendaciones}

Una de las principales limitaciones del presente trabajo es que los resultados de un único caso no son extrapolables ni generalizables a otras intervenciones con el mismo o similar diagnóstico. El presente trabajo, sin embargo, supone una propuesta metodológica centrada en la persona y basada en la ocupación que si puede ser extrapolable a otras intervenciones con este mismo diagnóstico o diagnósticos similares. En este sentido, resultaría muy valioso para la Terapia Ocupacional utilizar diseños de investigación en los que se pretendan recoger resultados de las intervenciones en términos de cambios en el desempeño ocupacional como oportunidad para incrementar el cuerpo de conocimiento de la ocupación como eje central del proceso de terapia ocupacional y de los diseños de investigación en terapia ocupacional.

Por otro lado, no se han monitorizado los resultados a medio/largo plazo. Dada la cronicidad de este diagnóstico y su curso en forma de brotes/remisiones, sería oportuno registrar posibles cambios funcionales en el tiempo y la respuesta/adaptación de la paciente. En este sentido, otra variable a medir tras la intervención podría ser el empoderamiento.

Finalmente, en relación con lo anterior, también sería oportuno registrar a medio/largo plazo el grado de "engagement" o compromiso con las ocupaciones que dotan de sentido a la vida, como factor predictor del bienestar personal y la calidad de vida, de las personas que tienen una condición de salud que supone una discapacidad crónica.

\section{CONCLUSIONES}

La intervención de terapia ocupacional centrada en la persona y basada en la ocupación supone no sólo cambios en el desempeño ocupacional, sino también en la calidad de vida percibida.
Cabe pensar, por otro lado, que estas intervenciones favorecen un mayor "engagement" ocupacional y, por tanto, un mayor bienestar personal. Es necesario profundizar en éstos aspectos tanto desde la perspectiva clínica como desde la investigadora.

Finalmente, el enfoque basado en la ocupación (topdown) versus otros enfoques tradicionales en rehabilitación centrados en la mejora de las capacidades funcionales (down-top), también logra resultados positivos no sólo en el desempeño ocupacional/funcionamiento sino también en las deficiencias en funciones corporales (dolor, perdida fuerza y limitación del rango de movimiento). Esto nos debe animar a desarrollar intervenciones e investigaciones centradas en la ocupación para hacer crecer el cuerpo de conocimiento de nuestra propia disciplina.

\section{Declaración de Financiación}

Este estudio carece de fuentes de financiación.

\section{Declaración de Conflicto de Intereses}

Los autores manifiestan no tener ningún conflicto de intereses.

\section{RefERENCIAS BibLIOGRÁfiCAS}

Bovend'Eerdt, T. J., Botell, R. E., \& Wade, D. T. (2009). Writing SMART rehabilitation goals and achieving goal attainment scaling: a practical guide. Clinical rehabilitation, 23(4), 352-361. doi:10.1177/0269215508101741

Christie, A., Jamtvedt, G., Dahm, K. T., Moe, R. H., Haavardsholm, E. A., \& Hagen, K. B. (2007). Effectiveness of Nonpharmacological and Nonsurgical Interventions for Patients With Rheumatoid Arthritis: An Overview of Systematic Reviews. Physical Therapy, 87(12), 1697-1715. doi:10.2522/ptj.20070039

Dziedzic, K., Nicholls, E., Hill, S., Hammond, A., Handy, J., Thomas, E., \& Hay, E. (2015). Self-management approaches for osteoarthritis in the hand: A $2 \times 2$ factorial randomised trial. Annals of the Rheumatic Diseases, 74(1), 108-118. doi:10.1136/annrheumdis-2013-203938

Ekelman, B. A., Hooker, L., Davis, A., Klan, J., Newburn, D., Detwiler, K., \& Ricchino, N. (2014). Occupational therapy interventions for adults with rheumatoid arthritis: An appraisal of the evidence. Occupational Therapy in Health Care, 28(4), 347-361. doi:10.310 9/07380577.2014.919687

Hammond, A., Young, A., \& Kidao, R. (2004). A randomised controlled trial of occupational therapy for people with early rheumatoid 
arthritis. Annals of the rheumatic diseases, 63(1), 23-30. doi:10.1136/ ard.2002.001511

Hervás, M. T., Navarro Collado, M. J., Peiró, S., Rodrigo Pérez, J. L., López Matéu, P., \& Martínez Tello, I. (2006). Versión Española del cuestionario DASH. Adaptación transcultural, fiabilidad, validez y sensibilidad a los cambios. Medicina Clinica, 127(12), 441-447. doi:10.1157/13093053

Kjeken, I. (2011). Occupational therapy-based and evidence-supported recommendations for assessment and exercises in hand osteoarthritis. Scandinavian Journal of Occupational Therapy, 18(4), 265-281. doi:10.3109/11038128.2010.514942

Kjeken, I., Dagfinrud, H., Slatkowsky-Christensen, B., Mowinckel, P., Uhlig, T., Kvien, T. K., \& Finset, A. (2005). Activity limitations and participation restrictions in women with hand osteoarthritis: Patients' descriptions and associations between dimensions of functioning. Annals of the Rheumatic Diseases, 64(11), 1633-1638. doi:10.1136/ard.2004.034900

Law, M., Baptiste, S., Mccoll, M., Opzoomer, A., Polatajko, H., \& Pollock, N. (1990). The Canadian Occupational Performance Measure: An Outcome Measure for Occupational Therapy. Canadian Journal of Occupational Therapy, 57(2), 82-87. doi:10.1177/000841749005700207

Lucas, R (1998). Versión española del WHOQOL. Madrid: Ediciones Ergon. Martínez Piédrola, M. R., Pérez de Heredia Torres, M., \& Gómez Calero, C. (2015). Terapia de la mano. Madrid: Síntesis.

McConnel, S., Beaton, D., \& Bombardier C. (1999). Disabilities of the arm, shoulder and hand: the DASH outcome measure user's manual. Toronto, Ontario: Institute for Work and Health.

Mease, P. J., \& Coates, L. C. (2018). Considerations for the definition of remission criteria in psoriatic arthritis. Seminars in arthritis and rheumatism, 6(47), 786-796. doi:10.1016/j.semarthrit.2017.10.021

Ottenbacher, K. J., \& Cusick, A. (1990). Goal attainment scaling as a method of clinical service evaluation. American Journal of Occupational Therapy, 44(6), 519-525. doi:10.5014/ajot.44.6.519

Riemsma, R. P., Taal, E., Kirwan, J. R., \& Rasker, J. J. (2004). Systematic review of rheumatoid arthritis patient education. Arthritis care \& research, 57(6), 1045-1059. doi:10.1002/art.20823

Siegel, P., Tencza, M., Apodaca, B., \& Poole, J. L. (2017). Effectiveness of occupational therapy interventions for adults with rheumatoid arthritis: A systematic review. American Journal of Occupational Therapy, 77(1), 1-11. doi:10.5014/ajot.2017.023176

Steultjens, E. M., Dekker, J., Bouter, L. M., Leemrijse, C. J., \& Van Den Ende, C. H. (2005). Evidence of the efficacy of occupational therapy in different conditions: an overview of systematic reviews. Clinical rehabilitation, 19(3), 247-254. doi:10.1191/0269215505cr870oa

Steultjens, E. M., Dekker, J., Bouter, L. M., Van Schaardenburg, D., van Kuyk, M. A. H., \& Van Den Ende, C. H. (2002). Occupational therapy for rheumatoid arthritis: a systematic review. Arthritis Care \& Research: Official Journal of the American College of Rheumatology, 47(6), 672-685. doi:10.1002/art.10801
WHOQOL Group. (1998). Development of the World Health Organization WHOQOL-BREF Quality Of Life assessment. Psychology Medicine, 28(3), 551-558.

World Federation of Occupational Therapists (2019). Occupational Therapy and Rehabilitation. Recuperado de www.wfot.org/ resources/occupational-therapy-and-rehabilitation

Zhang, W., Doherty, M., Leeb, B. F., Alekseeva, L., Arden, N. K., Bijlsma, J. W., ... Zimmermann-Górska, I. (2007). EULAR evidence based recommendations for the management of hand osteoarthritis: Report of a Task Force of the EULAR Standing Committee for International Clinical Studies Including Therapeutics (ESCISIT). Annals of the Rheumatic Diseases, 66(3), 377-388. doi:10.1136/ ard.2006.062091 
